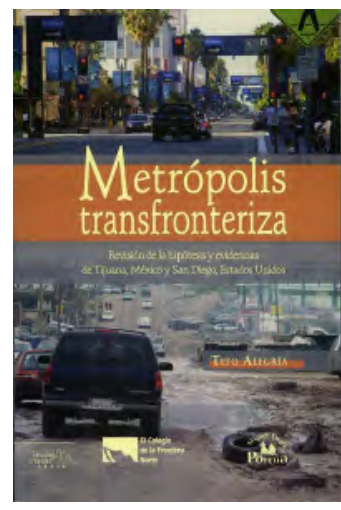

El objetivo del libro es evaluar si Tijuana y San diego son dos ciudades separadas o una sola área metropolitana. Para ello el autor echa mano de dos niveles de análisis. Primero, hace una comparación agregada de las estructuras económicas y poblacionales de ambas ciudades, en segundo lugar, hace una comparación micro de la forma urbana y los mecanismos generadores de forma. El análisis micro se enfoca en dos elementos: el uso del suelo residencial y la formación de centros terciarios. La investigación de Alegría genera suficiente información empírica y analítica que lo lleva a concluir con certeza que "Tijuana y San Diego son diferentes tanto en forma urbana como en sus mecanismos generadores de forma" y, por lo tanto, no constituyen una región metropolitana transfronteriza. Esta conclusión es muy importante, ya que marca una ruptura con 20 años de trabajos de investigación urbana en la Frontera Norte de México. Pero la relevancia de esta conclusión va más allá de los estudios urbanos. En primer lugar, plantea una posibilidad que afecta la comprensión de la frontera mexicana con los Estados Unidos y de otras fronteras en el mundo con condiciones similares. En segundo lugar, el
Metrópolis Transfronteriza. REVISIÓN DE LA HIPÓTESIS Y EVIDENCIAS de Tijuana, México, y San Diego, EstADOS UNIDOS

\section{Tito Alegría}

COLEF-PORRÚA, MÉXICO. 2009, 400 PP.

que un continuo urbano separado por una frontera internacional funcione como dos ciudades, plantea la necesidad de una reformulación de diversos planteamientos en los que se asume una integración funcional, en particular para los estudios fronterizos desde la economía y la sociología. Antes de desarrollar esta idea, describiré la argumentación y contenido de cada capítulo.

El primer capítulo se compone de dos partes. En la primera, se hace una revisión crítica de las teorías que se han generado para explicar el funcionamiento de las ciudades de Tijuana y San diego. Estas teorías desde diferentes disciplinas han apuntado hacia la noción de una región o una metrópolis transfronteriza. En un análisis detallado en el que se deconstruyen los postulados de diferentes autores, Alegría critica los supuestos subyacentes a cada una, algunas veces cuestionando su construcción lógica y en otros confrontando y aportando evidencia empírica. En la segunda parte de este primer capítulo, el autor analiza críticamente y en extenso las diferentes teorías y metodologías sobre la morfología urbana. Es un recorrido sobre los debates sobre la segregación espacial y los métodos para su análisis. 
En el segundo capítulo, se comparan procesos estructurales macro en las dos ciudades. Se inicia por comparar los procesos económicos encontrando que el crecimiento y especialización económica de San Diego no se puede conectar a factores de proximidad con la frontera, sino, por el contrario, a la formación de conglomerados de alta tecnología, mientras que Tijuana ha tenido un crecimiento económico determinado por su cercanía a la frontera. Por otra parte, complementa el análisis con los procesos demográficos y encuentra que las razones de los cambios en el crecimiento son diferentes para ambas ciudades. En la siguiente sección, Alegría cuestiona la noción de una sociedad transfronteriza, ya que ésta supone que los grupos sociales de ambos lados de la frontera presentan prácticas sociales comunes, a lo que confronta el hecho de que la mitad de la población tijuanense no puede cruzar la frontera y la mayoría de la población en San Diego no quiere cruzar a Tijuana, por lo que la interacción entre ambas poblaciones es mucho menor del que se asume y el conocimiento mutuo en sus praxis sociales es por consiguiente muy débil. Finalmente, analiza la posibilidad de que existiera un espacio transfronterizo urbano. En cuanto a la existencia de mercados transfronterizos, prueba contundentemente que no existen mercados unidos que formen una sola área transfronteriza, los precios y los componentes que los o influyen son distintos en cada ciudad.

En el capítulo 3 se analiza la hipótesis de una ciudad transfronteriza por medio de la estructura intraurbana también a nivel macro. Para llevarlo a cabo se utilizan los conceptos de "forma urbana" y los "mecanismos de generación” de esa forma. La hipótesis es que si se tratara de una misma ciudad, tendrían que presentar mecanismos similares de asignación de usos y, por lo tanto, usos similares. Después de un recorrido por los enfoques y metodologías del análisis de la estructura intraurbana -segregación y formación de subcentros- los datos generados y analizados por el autor señalan claramente la existencia de mecanismos diferenciados y, por lo tanto, de procesos de segregación sociorresidencial y formación de centros terciarios de forma diferente para San Diego y Tijuana.

En los siguientes capítulos el autor introduce el análisis micro de los procesos de generación de forma urbana en Tijuana y San Diego. En el capítulo 4 construye las hipótesis, desarrolla los modelos estadísticos y aclara el tipo de datos disponibles para cada ciudad. En el capítulo 5 se va probando cada modelo estadístico, primero los relacionados con los procesos de segregación y en seguida los relacionados con la formación de centros. Las conclusiones refuerzan los hallazgos a nivel macro: Tijuana y San Diego son ciudades independientes y no constituyen una zona metropolitana transfronteriza. En el último capítulo, Alegría sintetiza los hallazgos de su investigación y que fueron presentados a lo largo de los capítulos.

La conclusión de que son dos ciudades separadas es muy importante, ya que en los estudios urbanos fronterizos ha dominado la visión de metrópolis transfronterizas. La construcción argumental de una ciudad funcionalmente integrada pero separada por una frontera internacional ha sido muy atractiva, tanto desde el punto de vista político como académico. En términos políticos, el argumento de la existencia de problemas compartidos, de la soluciones de cooperación, de la unidad de los destinos, aporta un discurso de confianza en la integración a una sociedad desarrollada. Por el lado académico, Tijuana y su integración a San Diego, se presenta como una ventana privilegiada para observar los procesos más generales de globalización. La integración económica y la generación de un mismo espacio urbano-económico se muestra o se asume como dada en un proceso de evolución hacia estadios superiores de crecimiento económico y desarrollo endógeno. Alegría nos recuerda que la frontera cada vez es más restrictiva y que no ha evolucionado linealmente hacia estadios de mayor permeabilidad. Por el contrario, cada vez es menos la proporción de tijuanenses que puede cruzar la frontera, a la vez que cada vez menos sandieguinos quieren 
cruzarla. En este sentido, cada ciudad es cada vez más monocultural.

Mostrar con evidencia empírica que dos ciudades con áreas urbanas contiguas y que muestran procesos de intercambio intensos no forman un área metropolitana es complejo, tanto conceptual como metodológicamente. Alegría logra de forma clara generar evidencia, mejorando una serie de modelos y comparaciones estructurales. Lo importante es que desde otras disciplinas hubiera sido difícil probar la separación en los procesos fundamentales que definen la funcionalidad e integridad de una ciudad.

En particular, me parece importante la contribución y el reto que el trabajo de Alegría hace a la geografía económica en lo general y a los estudios económicos fronterizos en particular. Por una parte, contribuye al reforzar la visión institucionalista de las economías regionales. En última instancia lo que define a una economía regional en su trayectoria y su ritmo de cambio es el contexto institucional. Los contextos institucionales de San Diego y Tijuana caracterizan sus procesos internos y las diferencian en sus formas urbanas resultantes. Pero no sólo son las estructuras urbanas las que varían, son también las trayectorias económicas, que aunque con una gran integración, generan procesos y resultados distintos en cada ciudad.

A los geógrafos económicos y a los especialistas en desarrollo regional nos plantea un reto, ya que las nuevas teorías indican que los recursos económicos más relevantes en esta época de la globalización y la economía del conocimiento son los recursos intangibles, las convenciones y prácticas económicos que generan ventajas para sistemas aglomerados en una región. Nuevamente la cercanía de Tijuana y San Diego, y la existencia de una frontera internacional, parecería generar las condiciones para la existencia de convenciones comunes, para el paso irrestricto de prácticas económicas entre un lado y otro de la frontera. Sin embargo, por los resultados y especializaciones económicas de ambos lados sabemos que no está sucediendo. El trabajo de Alegría genera en este punto indicios de por qué no sucede esto, y es que la integración económica no va acompañada de la formación de procesos sociales que tiendan a homogeinizar; por el contrario, generan diferencia. En este sentido, los recursos intangibles (no basados en ventajas materiales como infraestructura o tecnología de producción) permanecen atados a cada ciudad, y como hemos visto se trata de dos ciudades y no de un área metropolitana transfronteriza.

Con lo anterior quiero señalar que las conclusiones de la investigación de Alegría deben de impactar más allá del ámbito estricto de del desarrollo urbano y del estudio de las ciudades fronterizas. El trabajo de este autor ha cruzado fronteras disciplinarias con anterioridad, y esperamos que nuevamente sus proposiciones generen puntos de discusión para entender el proceso fronterizo de una mejor forma. Seguramente lo hará, ya que la tesis del libro es controversial y generará cuestionamientos teóricos y metodológicos.

Finalmente quiero señalar que este libro es también una contribución práctica a la enseñanza de los temas de desarrollo urbano y de las ciudades fronterizas. Los capítulos teóricos y de revisión de la literatura ofrecen síntesis críticas de extensos cuerpos teóricos que no fácilmente son encontrados en español. Asimismo, la revisión de los modelos estadísticos para probar las diferentes hipótesis puede ser también utilizada como texto para la docencia. IEURE

\section{Alejandro Mercado Celis.}

Profesor Titular. UaM-Cuajimalpa,

Departamento de Ciencias Sociales. 